\title{
Author Correction: Tuning the interlayer spacing of graphene laminate films for efficient pore utilization towards compact capacitive energy storage
}

Zhuangnan Li (D), Srinivas Gadipelli (D), Hucheng Li, Christopher A. Howard (D), Dan J. L. Brett, Paul R. Shearing, Zhengxiao Guo (D), Ivan P. Parkin (D) and Feng Li $\mathbb{D}$

Correction to: Nature Energy https://doi.org/10.1038/s41560-020-0560-6, published online 17 February 2020.

In the version of this Article originally published, the corresponding e-mail address for Zhengxiao Guo was 'z.x.guo@ucl.ac.uk', but should have been 'zxguo@hku.hk', and affiliations 7, 8 and 9 incorrectly included the term "Present address:". These errors have been corrected in all versions.

Published online: 16 March 2020

https://doi.org/10.1038/s41560-020-0588-7

(C) The Author(s), under exclusive licence to Springer Nature Limited 2020 